\title{
Pancreatic islet dysfunction is partially attenuated by replacement of dietary saturated fatty acids with monounsaturated fatty acids
}

\author{
J.C. Ralston ${ }^{1}$, M.S. Nguyen-Tu' ${ }^{2}$, C.L. Lyons ${ }^{1}$, A.M. Murphy ${ }^{1}$, A.A. Cooke ${ }^{1}$, A. Falvey ${ }^{1}$, \\ O.M. Finucane ${ }^{1}$, G.A. Rutter ${ }^{2}$ and H.M. Roche ${ }^{1}$ \\ ${ }^{1}$ School of Public Health, Physiotherapy and Sports Science, University College Dublin, Dublin, Republic of Ireland and \\ ${ }^{2}$ Department of Medicine, Imperial College London, Hammersmith Hospital, W12 ONN, London, United Kingdom
}

Previous work has established that different dietary fats can have varying impacts on metabolism and insulin sensitivity ${ }^{(1)}$. Specifically, saturated fatty acids (SFA) drive insulin resistance and inflammation, while dietary monounsaturated fatty acids (MUFA) do not disrupt metabolism to the same extent ${ }^{(2)}$. Moreover, feeding a high-fat diet (HFD) enriched with MUFA, rather than SFA-HFD, causes less IL-1 $\beta$ mediated inflammation, insulin resistance and hyperinsulinemia ${ }^{(3)}$. Our aim was to establish whether switching to a MUFA-based diet after previously feeding a SFA-based diet could ameliorate hyperinsulinemia, inflammatory markers and pancreatic islet function.

A total of 30 male C57BL/J6 mice were assigned one of three diets: i) a low fat diet for 32 weeks (LFD; $10 \% \mathrm{kcal} ; \mathrm{n}=10$ ), ii) a SFA-based HFD for 32 weeks (SFA-HFD; $45 \% \mathrm{kcal} ; \mathrm{n}=10$ ) or iii) a SFA-based diet for 16 weeks followed by a MUFA-based diet for an additional 16 weeks (MUFA-HFD; $45 \% \mathrm{kcal} ; \mathrm{n}=10$ ). Fasting glucose and insulin levels were used to calculate HOMA-IR and HOMA- $\% \beta$. Glucose-stimulated insulin secretion response $(1.5 \mathrm{~g} / \mathrm{kg}$ intraperitoneal glucose) was assessed at baseline and following the 32-week diets. Following the diets, mice were killed and metabolic tissues (e.g., liver, adipose tissue, and pancreas) were harvested. Pancreatic islets were isolated by collagenase digestion and Ficoll separation, and were subsequently used to assess glucose-stimulated insulin secretion. Islet gene expression was analyzed by real-time RT-PCR, and pancreatic immunostaining was used to assess expression of insulin, IL-1 $\beta$ as well as macrophage infiltration. Statistical analysis included 1-way or 2-way ANOVA, where appropriate, with Bonferroni post-hoc comparisons.

HOMA-IR was elevated in both SFA-HFD and MUFA-HFD groups; however, the degree of insulin resistance was significantly lower in the MUFA-HFD group at 20, 24 and 32 weeks. Furthermore, the MUFA-HFD group exhibited reduced hyperinsulinemia compared to the SFA-HFD group $(P<0.001)$. Islets isolated from the SFA-HFD group had a significantly lower $(\sim 50 \%, P=0.029)$ insulin stimulatory index compared to the LFD group. Interestingly, this decline was partially reduced in the MUFA-HFD group. Pancreatic macrophage infiltration and IL-1 $\beta$ content were higher in the SFA-HFD group compared to both the LFD and MUFA-HFD groups. Finally, gene expression analysis of markers of $\beta$-cell development and function (e.g., insulin, Nkx6.1, Pdkl) revealed significant declines in the SFA-HFD group compared to the LFD and MUFA-HFD groups $(27 \%, 36 \%$, and $15 \%$, respectively). Ampk expression was also reduced in islets isolated following the SFA-HFD but was preserved in MUFA-HFD islets. Moreover, there was a $57 \%$ reduction in $I L-1 \beta$ in islets from the MUFA-HFD group compared to both LFD and SFA-HFD fed mice. In conclusion, this work suggests that changing from a SFA-HFD to a MUFA-HFD can partially prevent pancreatic dysfunction, and emphasizes the varying effects of different dietary fats on metabolic health.

1. Murphy AM, Lyons CL, Finucane OM et al. (2015) Prostaglandins Leukot Essent Fatty Acids 92, 49-55.

2. Lyons C, Kennedy EB \& Roche HM (2016) Nutrients 8(5), 247-268.

3. Finucane, Lyons CL, Murphy AM et al. (2015) Diabetes 64(6), 2116-2128. 